\title{
PARECERISTAS Ad Hoc 2017
}

Adriana Varani - UNICAMP

Adriano Toledo Paiva - UEMG

Aguinaldo Rodrigues Gomes - UFMT

Alessandra Elizabeth F. Gomes Prado UFSCar

Alessandra R. de Almeida - PUC/Campinas.

Aline Aparecida Veltrone - SME/Campinas-SP

Ana Lúcia Félix dos Santos - UFPE

Ana Valéria de Figueiredo da Costa - UERJ

Andreza Schiavoni - UEL

Angélica da Fontoura Garcia Silva - Anhanguera

Aparecida Rodrigues Silva Duarte - UNIVÁS

Artur Renda Vitorino - PUC/Campinas

Betânia de Oliveira Laterza Ribeiro - UFU

Camila Itikawa Gimenes - UFPR

Carla Helena Fernandes - UNIVÁS

Carolina de Roig Catini - UNICAMP

Cristiane Machado - UNICAMP

Débora Dainez - UFSCar

Desire Luciane Dominschek -

Eli Andrade Rocha Prates - UNASP

Elizabete Maria Espíndola - UNIVÁS

Emília Carvalho Leitão Biato - UNB

Enio Antônio de Almeida - Anhanguera

Eraldo Leme Batista

Fabiana de Cássia Rodrigues - UNICAMP

Fábio Abreu dos Passos UFPI

Fernanda de O. S.Taxa PUC/Campinas

Flavia Sueli Fabiani Marcatto - UNIFEI

Giovana Azzi de Carmargo - UNIFIA

Graziela Giusti Pachane - UFTM

Gustavo Cunha de Araújo - UFT

Helena Ribeiro de Castro - Instituto Piaget/PT

Jaqueline Andrade Calixto - UFU

Jefferson Carriello do Carmo - UNISO

João Baptista de Almeida Junior Unisal

Jocemara Ferreira Mognon - UNIBRASIL

José Carlos Araújo - UFU

Juliano Peroza - IFPR/Irati

Laura Noemi Chaluh - UNESP/Rio Claro

Leonardo Rocha de Almeida- Unilasalle/RS

Lilian do Rocio Borba - UNICAMP

Lisandra Borges Vieira Lima - USF

Luana Ferrarotto - IFSP/Bragança

Luciana Cristina Salvatti Coutinho - UFSCar

Manoel Araujo Teixeira - UNIVÁS
Marcele Frossard de Araujo - UERJ

Marcos Antônio Batista - UNIVÁS

Margarida Montejano da Silva - SME/Campinas

Maria Adélia Costa - CEFET/MG

Maria Adélia da Costa - CEFET/MG

Maria Cristina P. Innocentini Hayashi - UFSCar

Maria José Rosário - UFPA

Maria Márcia Sigristi Malavasi - UNICAMP

Maria Patrícia Freitas Lemos - UFPI

Maria Simone F. Pereira Moreira Costa - UFU

Mariana Veríssimo S. Aguiar e Silva- PUC/MG

Milta Neide Freire Barron Torrez - Fiocruz

Mônica Cristina M. de Moraes - PUC/Campinas

Nayane Martoni Piovezan FAE C. Universitário

Neide Pena Cária - UNIVÁS

Nielce Meneguelo Lobo da Costa - UNIAM

Nilo Agostini - USF

Olinda Maria Noronha - UNICAMP

Otavio Henrique Ferreira da Silva - UFMG

Patrícia Cardoso M.do Amaral Araujo - UFRJ

Patrícia Vieira Trópia - UFU

Raquel Baptista Spaziani UNIB

Renata Maria Z. Roamnholi - UNESPE/Botucatu

Rita de Cássia Silva Godoi Menegão - UFMT

Rodrigo Pelloso Gelamo - UNESP/Marília

Rodrigo Sarruge Molina PUC/Campinas

Rosana Maria Mohallem Martins - FEPI

Rosane de Fátima Batista Teixeira - IFPR

Rosemary Rodrigues Oliveira UNESP/Jaboticabal

Rosimeri Silva Pereira - UFRJ - UNIRIO

Rosinalda Aurora de Melo Teles - UFPE

Sandra Mara Ferreira - SEED/PR

Sarah Maria de Freitas M. Silva - UNIVÁS

Simão Pedro Pinto Marinho - PUC/MG

Sônia Aparecida Siquelli - UNIVÁS

Tânia Barbosa Martins - UNIMEP

Tânia Regina Lobato dos Santos - UEPA

Thaís Carvalho Z. Penteado - SME/Campinas

Thatiana Helena de Lima - UFBA

Valmir Pereira - UEPB

Vânia dos Santos Mesquita - UNIVÁS

Vera Lúcia de Carvalho Machado - PUC/Campinas

Vera Lucia Martiniak - UEPG

Weder Ferreira da Silva - UFF 